\title{
Neuroendocrine Neoplasms: Dichotomy, Origin and Classifications
}

\author{
Günter Klöppel \\ Consultation Center for Pancreatic and Endocrine Tumors, Institute of Pathology, Technical University München, Munich, Germany
}

\section{Keywords}

Neuroendocrine neoplasms · Heterogeneity · Origin ·

Classification - Gut · Pancreas

\section{Summary}

Neuroendocrine neoplasms (NENs) are heterogeneous tumors with a common phenotype. There are two fundamentally different groups of NENs: well-differentiated, low-proliferating NENs, called neuroendocrine tumors (NETs) or carcinoids, and poorly differentiated, highly proliferating NENs, called small- or large-cell neuroendocrine carcinomas (NECs). This NEN dichotomy is probably due to an origin from different neuroendocrine progenitor cells. The current World Health Organization (WHO) classification of gastrointestinal NENs uses the Ki67 proliferation index to grade NETs as G1 or G2, and NECs as G3. In the pancreas, NETs and NECs may overlap in their proliferation index, making the distinction between them difficult and leading to therapeutic uncertainties. Therefore, the WHO classification of pancreatic NENs (PanNENs) from 2017 introduced a new NET G3 category. Helpful for the distinction of NETs G3 from NECs is the expression of p53 and rb1 that is usually negative in PanNETs. Comparison of the WHO classification of digestive system NENs with other NEN classifications reveals site-specific differences in terminology and a general lack of grading systems. However, all classifications recognize the existence of the two major NEN families and provide a general basis for their prognostic and therapeutic stratification. A development of a common NEN classification across organs is desirable.

\section{Introduction}

Neuroendocrine neoplasms (NENs) are something special. They seem to be a fairly homogeneous group but at a closer look they present as heterogeneous neoplasms with great differences in origin and biology. NENs occur in almost every organ or region of the body and originate from cells with a neuroendocrine phenotype. They share the expression of general neuroendocrine markers such as synaptophysin and chromogranin A but are diverse in terms of special histologic features, proliferation, hormonal production, molecular profile, and clinical aggressiveness. Therefore, their classifications vary by organ and type. Among these, the classification of the gastroenteropancreatic NENs (GEP-NENs) plays a central role since the digestive system, that harbors $70 \%$ of all NENs, provides a grading system, which the other classifications are lacking (table 1).

This review focuses in its first part on a general description of the NENs and the neuroendocrine cell system. In its second part, it discusses the morphological and molecular heterogeneity of NENs which results in a general dichotomy, distinguishing between well and poorly differentiated NENs. As the available data mainly derive from studies in pancreatic NENs (PanNENs), these tumors serve as a model for the dichotomy of the NENs. In its third part, this review article emphasizes the differences and commonalities of NENs throughout the body and presents a comparison of the current WHO classifications of the most important organ systems.

\section{Neuroendocrine Neoplasms and the Neuroendocrine} Cell System

Neuroendocrine neoplasms occur almost everywhere in the body but are most common in the gastrointestinal tract, the pancreas, and the lungs [1]. They are composed of cells that secrete cell type-specific peptide hormones and/or biogenic amines, which are stored in electron-dense membrane-bound granules. In addition,

\section{KARGER}

() 2017 S. Karger GmbH, Freiburg

Fax +497614520714
Prof. em. Dr. Günter Klöppel

Consultation Center for Pancreatic and Endocrine Tumors

Institute of Pathology, Technical University München

Ismaninger Straße 22, 81675 München, Germany

guenter.kloeppel@ tum.de 
Table 1. Site-specific relative frequency of neuroendocrine neoplasms in the body

\begin{tabular}{ll}
\hline Organ system & Proportion, \% \\
\hline Gastroenteropancreatic system & 70 \\
Respiratory system & 25 \\
Other primary sites & 5 \\
\hline
\end{tabular}

these cells express markers such as synaptophysin and chromogranin A that are largely independent of cell-specific hormone production. These general marker proteins reside in the membranes of intracytoplasmic small presynaptic-like vesicles (synaptophysin) or large dense core hormone granules (chromogranin A). The peptide hormone-producing cells share these markers with neuronal cells and have therefore received the label 'neuroendocrine'. Historically the term 'neuroendocrine' also refers to the proposed relationship of neural/neuroectodermal structures with Masson's silver-stained intestinal cells, Feyrter's clear cells, and Pearse's APUD cells [2-6].

The neuroendocrine cells are either diffusely distributed as 'individuals' in mucosal membranes, particularly in those of the digestive system, or are forming organs or organized cell clusters with endocrine function such as the pituitary, parathyroids, pancreatic islets, and paraganglia. They are thought to be either of epithelial or neuroectodermal origin. While the cells deriving from the neuroectoderm colonize in the various paraganglia and the olfactory membrane, the cells with epithelial features mainly occur in the mucosa of the gastrointestinal tract and the bronchial system, the thyroid (as C-cells), the parathyroids, and the pancreatic islets. The potential cells of origin of the epithelial neuroendocrine cells are local pluripotent (secretory) stem cells within the mucosal and glandular epithelium [1].

The epithelial NENs have been described under the terms of carcinoid, argentaffinoma, APUDoma, small cell carcinoma, islet cell tumor, medullary thyroid carcinoma, Merkel cell tumor, neuroendocrine tumor (NET), and neuroendocrine carcinoma (NEC). The neoplasms with neuroectodermal origin include paragangliomas, pheochromocytoma-neuroblastomas, and olfactory neuroblastomas. As all NENs (except pulmonary small cell carcinomas) are rare and, in addition, show site-specific differences in morphology, hormone production, and natural course, the assessment of their prognosis and response to treatment proved to be difficult for a long time.

In recent years, the treatment of NENs has been very much improved. This is the result of the introduction of novel drugs (such as somatostatin analogs), molecular therapies, increasing use of isotopic compounds that bind to receptors on neuroendocrine cells (somatostatin receptor-coupled radioligands), and sophisticated surgery or liver-directed locoregional approaches [7, 8]. Essential for this progress was the evolution of the classification of GEPNENs over the last two decades, which introduced reproducible criteria of prognostic significance. This evolution started in 1995 with the 'Munich classification' [9], as it was the backbone for the subsequent WHO classifications from 2000 and 2004 [10, 11] and the driving force for the staging and grading system proposed by the European Neuroendocrine Tumor Society (ENETS) in 2006 and 2007 [12, 13], and culminated in the 2010 and 2017 WHO classifications $[14,15]$. The latter depict the main differences in the morphological appearances of the GEP-NENs and their variable proliferative activity $[15,16]$.

As neuroendocrine cells do not only occur in the digestive system but also at other sites of the body, such as the respiratory tract or the prostate, NENs do also arise outside the digestive system and have been recorded, though mostly in low frequency, in almost all parts of the body. These NENs basically resemble the GEP-NENs regarding their morphologic differentiation and growth properties. Thus, well-differentiated, slowly growing NENs can be distinguished from poorly differentiated, fast growing NENs throughout the body, suggesting that the development of these two broad categories of neoplasms follows comparable tumorigenic pathways.

\section{Diversity of Neuroendocrine Neoplasms with Emphasis on Gastrointestinal and Pancreatic Neuroendocrine Neoplasms}

The diversity of NENs has been particularly well studied and classified in the gastroenteropancreatic system and the lung [17]. In this article, the focus lies on GEP-NENs. In the current WHO classifications (2010 for all GEP-NENs [14], 2017 only for PanNENs [15]), the NENs showing a well-differentiated histology and a low proliferation rate have been called NETs, while those NENs displaying a poorly differentiated histology and high proliferation rate are called NECs $[14,15]$. NETs are generally rather indolent, slowly growing neoplasms that produce peptide hormones or biogenic amines, that may be associated with hormonal syndromes (and are then called insulinoma, glucagonoma, gastrinoma, etc.), that express somatostatin receptors (particularly SSTR2A) [18, 19] (fig. 1a), and that are associated with hereditary tumor syndromes such as multiple endocrine neoplasia (MEN1) [20] or von HippelLindau syndrome (VHL) [21]. In contrast, NECs are aggressive, fast growing neoplasms that usually fail to express hormones or produce hormonal syndromes, and are not associated with hereditary tumor diseases (fig. 1b). This morphologic and biologic dichotomy which suggests two distinctly different NEN entities is further supported by data from recent genetic studies in PanNENs [22-24]. In sporadic well-differentiated PanNENs, i.e. PanNETs, the most common mutational alterations involve the MEN1 gene (in about 35-40\%), the mutually exclusively mutated DAXX (death-domain associated protein) or ATRX (alpha-thalassemiamental retardation syndrome) genes (in about 40\%), and the mTOR pathway genes (in about 15\%), in which DEPDC5 mutations are mutually exclusive to PTEN and TSC2. Moreover, DAXX/ $A T R X$ mutations appear to promote alternative lengthening of telomeres and chromosomal instability $[25,26]$. Epigenetic mechanisms rather than point mutations seem to alter the VHL gene [26]. KRAS, TP53, SMAD4, and p16, the driver genes of pancreatic ductal adenocarcinoma, are very rarely affected in well-differentiated PanNENs [26]. Conversely, poorly differentiated PanNENs frequently have TP53 and $R b 1$ mutations but lack gene alterations of the MEN1, DAXX/ATRX, and mTOR genes [19, 23]. 
Fig. 1. Histology of pancreatic neuroendocrine neoplasms (NEN). a Well-differentiated NEN showing a trabecular pattern and $\mathbf{b}$ an extensive immunolabeling of the cell membranes for SSTR2A. c Poorly differentiated NEN showing diffusely arranged cells and a small necrosis (bottom). d Abortive immunolabeling for SSTR2A.

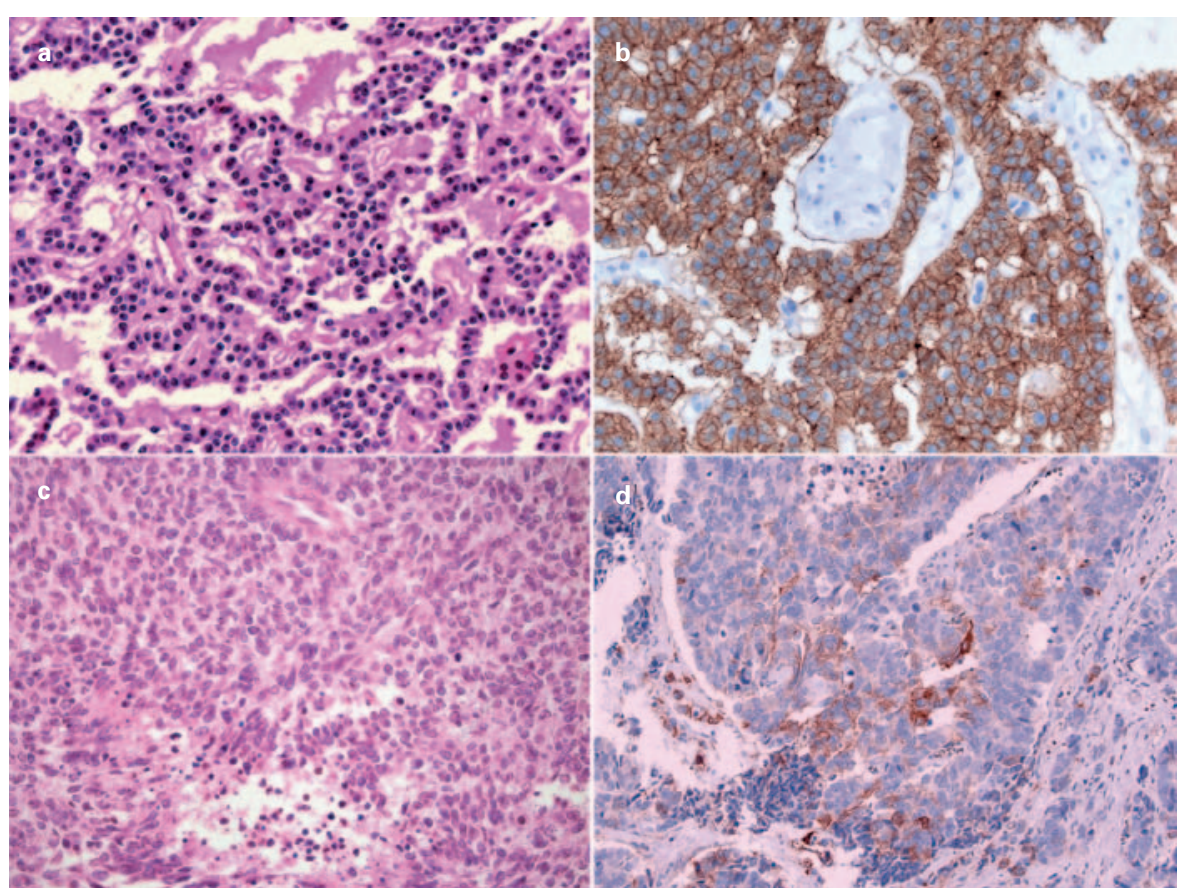

Support for the fundamental dichotomy of PanNENs also comes from observations in primary PanNETs (or their liver metastases developing during the course of the disease). It has been noted that PanNETs, which are defined by the 2010 WHO classification by a Ki67 index that should not exceed 20\% [19,27], may present, though rarely, with a Ki67 index greater than 20\%, and thus qualify, according to the 2010 WHO criteria, for a PanNEC. However, as these well-differentiated, but grade-discordant PanNETs were found to retain their typically well-differentiated histology and functional capacity, to maintain their genetic signature by showing MEN1 and DAXX/ARTX mutations, and to lack changes in expression and mutation of TP53 and Rb1, the new WHO classification from 2017 added a G3 PanNET category, defined by a Ki67 index greater than 20\%, to the existing PanNEN categories [15]. G3 PanNETs thus overlap in their proliferation activity with PanNECs but belong to the NET family in terms of morphology, biology, and genetics. Clinically, the introduction of a G3 PanNET category is important because these usually show a poor response to first-line platinum-based chemotherapy which PanNECs receive, while they respond favorably to surgery for resectable disease and to somatostatin analogs, evorolimus, sunitinib, and temozolomide in metastatic disease [28-30].

Although the available morphologic, genetic and clinical data, as discussed above, are consistent with the concept of two profoundly distinct NENs in the pancreas, the possibility that some rare poorly differentiated PanNENs might derive from well-differentiated PanNENs through a process of dedifferentiation should not be totally excluded. In individual cases of PanNETs it might happen that subclones develop (particularly in liver metastases during the course of the disease) which acquire genetic changes and phenotypical features similar to those of PanNECs.

\section{Origin of Pancreatic Neuroendocrine Neoplasms}

The molecular dichotomy observed in PanNENs, which has also been described in pulmonary NENs [31], suggests that well and poorly differentiated NENs develop along different pathogenetic pathways and could therefore originate from different types of precursor cells (fig. 2). In the gut and pancreas, embryological studies have shown that neuroendocrine cells derive from endodermal (secretory) stem cells under the control of certain transcription factors such as MATH1, PDX1, CDX2, NGN3, and ISL1 [1, 32, 33]. Studies in well-differentiated pancreatic NENs revealed that there is a differential expression of these transcription factors, depending on the dominant hormone expression in the tumors [34]. It is therefore likely that the mutational events which transform developing endodermal cells into NET cells with the help of transcription factors only affect those cells which have already started their program to produce a certain hormone.

Further support for the assumption that hormonally programmed cells give rise to NETs comes from studies on the development in MEN1-associated NETs in the pancreas and duodenum. In these organs, MEN1 is characterized by the occurrence of multiple tiny NETs (microadenomas) that are obviously the precursors to single large NETs which usually develop in the course of the disease [35-38]. By analyzing the development of these MEN1 tumors, using immunohistochemistry combined with the fluorescence in situ hybridization (FISH) test, it was found that some hormonally programmed neuroendocrine cells, predominantly expressing glucagon in the pancreas and gastrin in the duodenum, focally increase in number and show hyperplasia. The hyperplastic cells that, like all cells in the body, carry the MEN1 germ cell mutation on one allele of the MEN1 gene that resides in the 11q13 re- 
Table 2. Site-specific distribution of well and poorly differentiated neuroendocrine neoplasms (NENs) in relation to their relative frequency

\begin{tabular}{lll}
\hline Organ & $\begin{array}{l}\text { Well differentiated } \\
\text { NENs }\end{array}$ & $\begin{array}{l}\text { Poorly differentiated } \\
\text { NENs }\end{array}$ \\
\hline Pituitary & common & very rare \\
Thyroid & common & very rare \\
Parathyroid & common & very rare \\
Thymus & common & rare \\
Lung & rare & common \\
Pancreas & common & very rare \\
Esophagus & very rare & common \\
Stomach & common & rare \\
Small bowel & common & rare \\
Appendix & common & very rare \\
Colon & very rare & common \\
Rectum & common & rare \\
Urogenital organs & very rare & common \\
Skin & very rare & common \\
\hline
\end{tabular}

gion of chromosome 11, showed no allelic loss in this region when tested by FISH. However, this loss of heterozygosity, which is typically observed in advanced MEN1 tumors, was detected when the cell aggregates tested showed monoclonality and monohormonality, characterizing them as microadenomas. This indicates that in the special condition of MEN1, hormonally programmed neuroendocrine cells are able to transform into monoclonal NET cells by only one genetic event, i.e. the activation of the germline MEN1 mutation by loss of the MEN1 gene locus on the second allele [39]. If a similar sequence of morphologic and genetic changes is also responsible for the development of sporadic NETs in the pancreas, duodenum, or elsewhere is not known yet.

Precursor lesions in poorly differentiated NENs (NECs) have not been identified so far. These NENs do not seem to occur in MEN1 patients, neither in the pancreas nor in the lung. Their development can therefore not be studied in this condition, and potential cells of origin are difficult to define. Because of the different genetic profiles of NETs and NECs, however, their cells of origin presumably also differ. Instead from hormonally programmed neuroendocrine cells, NECs could derive from pluripotential (secretory) stem cells that are already committed to the neuroendocrine cell lineage ('primitive neuroendocrine precursor cell'), but are not yet hormonally defined [1]. If these still primitive neuroendocrine cells are tackled by mutational hits, basic genetic alterations are probably initiated, such as TP53, Rb1, and/or $p 16$ mutations that interfere with the normal cell cycle control and produce highly aggressive neoplastic cells. Their primitive neuroendocrine phenotype is characterized by the expression of general neuroendocrine markers and premature hormone granules but still lacks any programming and expression of definite hormonal products.

Whether the small and large cell subtypes of poorly differentiated NENs represent different subtypes, as suggested by their histology, is unclear so far. According to recent studies in pancreatic NENs they seem to be genetically related. Both subtypes were

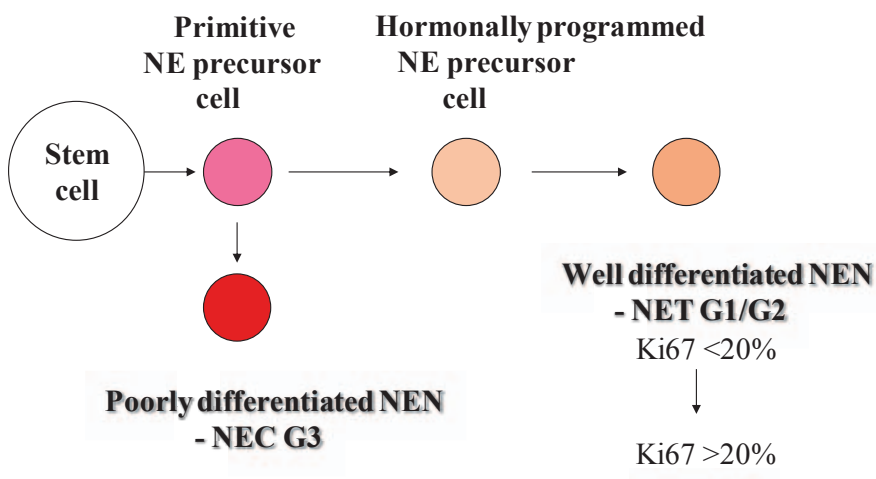

NET G3

Fig. 2. Cellular origin of pancreatic neuroendocrine neoplasms.

found to carry in equal frequency $T P 53, R b 1$, and $p 16$ mutations and BCL-1 expression [23]. However, as small and large cell subtypes often differ in their proliferative activity (the large cell subtypes usually show a lower Ki67 index) and their association with nonneuroendocrine elements such as adenocarcinoma components, it is conceivable that more detailed genetic studies might reveal some subtle differences between the two subtypes in the future.

\section{Site-Specific Heterogeneity of Neuroendocrine Neoplasms}

The strong morphologic similarities between GEP-NENs and extragastroenteropancreatic NENs suggest that the concept of two NEN families also applies to the NENs outside of the gastroenteropancreatic system. Using the terms carcinoid and (high-grade) NEC, NENs of both types have been described in numerous organs outside of the gastroenteropancreatic-neuroendocrine cell system. Most frequently they are observed in the lung, followed by the upper airways and other head and neck regions. In some organs, special terms are applied such as medullary carcinoma in the thyroid or Merkel cell carcinoma in the skin.

Despite the commonalities of the NENs, there are striking sitespecific features of the tumors. For instance, in the group of the GEP-NENs, there are subtle differences between ileal, appendiceal, and pancreatic NETs. Ileal and appendiceal NETs, though sharing the production of serotonin, differ fundamentally in their clinical course, which is largely benign in appendiceal NETs and usually malignant in ileal NETs. In contrast, ileal NETs follow a more indolent course than PanNETs and differ profoundly in their molecular profile from PanNETs. While the latter tumors are characterized by MEN1 and DAXX/ARTX alterations, the former tumors frequently show a loss of chromosome 8 and no driver mutations [40].

Other site-specific differences among the NENs concern the ratio of well-differentiated to poorly differentiated NENs. Welldifferentiated NENs clearly prevail in the pituitary, thyroid, para- 
Table. 3. Comparison of the current WHO classifications of neuroendocrine neoplasms of the pancreas, gastrointestinal tract, lung, head and neck, urogenital organs, and breast

\begin{tabular}{|c|c|c|c|c|c|c|}
\hline Pancreas, 2017 & $\begin{array}{l}\text { Gastrointestinal } \\
\text { tract, } \\
2010\end{array}$ & $\begin{array}{l}\text { Lung, } \\
\text { thymus, } \\
2015\end{array}$ & $\begin{array}{l}\text { Head and } \\
\text { neck, } \\
2016\end{array}$ & $\begin{array}{l}\text { Urological } \\
\text { organs, } \\
2016\end{array}$ & $\begin{array}{l}\text { Genital } \\
\text { organs, } \\
2014\end{array}$ & $\begin{array}{l}\text { Breast, } \\
2012\end{array}$ \\
\hline $\begin{array}{l}\text { NET G1, } \\
\quad \text { Ki67 <3\% }\end{array}$ & $\begin{array}{l}\text { NET G1 } \\
\text { Ki67 } \leq 2 \%\end{array}$ & $\begin{array}{l}\text { typical } \\
\text { carcinoid }\end{array}$ & $\begin{array}{l}\text { well differentiated } \\
\text { NEC }\end{array}$ & $\begin{array}{l}\text { well differentiated } \\
\text { NET }\end{array}$ & $\begin{array}{l}\text { carcinoid tumor } \\
\text { (cervix, uterus) }\end{array}$ & $\begin{array}{l}\text { NEC, well } \\
\text { differentiated }\end{array}$ \\
\hline $\begin{array}{l}\text { NET G2, } \\
\quad \text { Ki67 3-20\% }\end{array}$ & $\begin{array}{l}\text { NET G2 } \\
2-20 \%\end{array}$ & $\begin{array}{l}\text { atypical } \\
\text { carcinoid }\end{array}$ & $\begin{array}{l}\text { moderately } \\
\text { differentiated NEC }\end{array}$ & & $\begin{array}{l}\text { atypical carcinoid } \\
\text { tumor (cervix) }\end{array}$ & \\
\hline $\begin{array}{l}\text { NEC, } \\
\text { Ki67 >20\%, } \\
\text { small cell } \\
\text { type }\end{array}$ & $\begin{array}{l}\text { NEC > 20\%, } \\
\text { small cell type }\end{array}$ & $\begin{array}{l}\text { NEC, small } \\
\text { cell type } \\
\text { (also combined } \\
\text { small cell NEC) }\end{array}$ & $\begin{array}{l}\text { poorly } \\
\text { differentiated NEC } \\
\text { (small cell) }\end{array}$ & small cell NEC & $\begin{array}{l}\text { small cell } \\
\text { carcinoma, } \\
\text { pulmonary type; } \\
\text { (ovary) } \\
\text { small cell NEC } \\
\text { (vagina, cervix, } \\
\text { uterus) }\end{array}$ & \\
\hline $\begin{array}{l}\text { NEC, } \\
\text { Ki67 >20\%, } \\
\text { large cell } \\
\text { type }\end{array}$ & $\begin{array}{l}\text { NEC }>20 \% \text {, } \\
\text { large cell type }\end{array}$ & $\begin{array}{l}\text { NEC, large } \\
\text { cell type } \\
\text { (also combined } \\
\text { large cell NEC) }\end{array}$ & $\begin{array}{l}\text { poorly } \\
\text { differentiated NEC } \\
\text { (large cell) }\end{array}$ & large cell NEC & $\begin{array}{l}\text { large cell } \\
\text { NEC (vagina, } \\
\text { cervix, uterus) }\end{array}$ & $\begin{array}{l}\text { NEC, poorly } \\
\text { differentiated } \\
\text { (small cell } \\
\text { carcinoma) }\end{array}$ \\
\hline MiNEN & MANEC & $\begin{array}{l}\text { combined } \\
\text { small cell NEC }\end{array}$ & & $\begin{array}{l}\text { adenocarcinoma } \\
\text { with neuroen- } \\
\text { docrine differentia- } \\
\text { tion (prostate) }\end{array}$ & & $\begin{array}{l}\text { carcinoma with } \\
\text { neuroendocrine } \\
\text { differentiation }\end{array}$ \\
\hline
\end{tabular}

NET = Neuroendocrine tumor; NEC = neuroendocrine carcinoma; MiNEN = mixed neuroendocrine-nonneuroendocrine neoplasm; MANEC $=$ mixed adeno-neuroendocrine carcinoma thyroid, thymus, stomach, small bowel, appendix, rectum, and pancreas, while poorly differentiated NENs are relatively more common in the lung, esophagus, colon, urogenital organs, and skin (table 2). It is also of interest to study the distribution of mixed neuroendocrine-nonneuroendocrine neoplasms (MiNENs) in the body. They are common in the stomach, colon, lung, prostate, and probably also in the uterus, but rare in the pancreas as well as in the head and neck region.

Looking at the different incidence figures of the various NEN types in the body, the question arises whether this is related to the causes of these neoplasms. In sporadic well-differentiated NENs, the causes are not known and may be related to unpredictable chromatin remodeling and instability, or may be caused by germline gene mutations underlying familial and hereditary tumor syndromes such as MEN1 or VHL. In poorly differentiated NENs, however, there are well-known causes. Thus, smoking is strongly associated with small cell lung NECs (and probably also with urinary bladder NENs), and many Merkel cell carcinomas are related to polyomavirus (MCPyV) infection or ultraviolet irradiation. Environmental factors could also play a role in poorly differentiated GEP-NENs, as it is striking that these neoplasms predominantly occur in the stomach and colon where extrinsic carcinogens are thought to play an etiological role in carcinoma development. Conversely, well-differentiated NENs are most prevalent in gastrointestinal regions such as the small intestine and the appendix where environmentally driven carcinomas are very rare.

\section{Current Classifications of Neuroendocrine Neoplasms}

Although the NENs from the different regions of the body share most of their morphologic and biologic features, their classifications differ more or less in terminology and stratifying criteria. However, common to all these classifications is the recognition of two NEN families: the well-differentiated NENs (including also moderately differentiated NENs) and the poorly differentiated NENs (table 3). The terms that are used for the well-differentiated NENs include carcinoid, NETs, and well-differentiated NECs. For the poorly differentiated NENs the names small cell or large cell NEC are generally used.

The classifications that are currently most refined regarding the recognition of the diversity of the neoplasms including their prognosis are the WHO classification of GEP-NENs from 2010 and the 2017 classification that only covers the PanNENs. These classifications use the label 'neuroendocrine neoplasm' in order to encompass all tumors and carcinomas of the neuroendocrine cells, regardless of their differentiation and biology. If neuroendocrine neoplastic cells combine with nonneuroendocrine (usually adenocarcinoma) cells, the resulting neoplasms are labelled 'mixed' tumors. Moreover, these classifications are based on the assumption that all GEP-NENs have some malignant potential. Finally, these classifications define a three-tiered grading system based on mitotic count and/or Ki67 index whose strong prognostic value has been repeatedly validated [41]. As a result, they basically divide the NENs into three groups: i) the well-differentiated NENs which are 
called NETs, ii) the poorly differentiated NENs, called NECs of small and large cell type, and iii) the mixed adeno-neuroendocrine carcinomas or, in case of the PanNENs, the MiNENs. The NETs are then subdivided by their proliferative activity, using the mitotic and/or Ki67 index, into either G1 NETs or G2 NETs, and in case of the PanNETs, also into G3-NETs. The NECs are by definition G3 carcinomas (table 3). The mixed neoplasms, where each component has to exceed $30 \%$ of the neoplastic cells, are mostly composed of a NEC G3 that combines with a poorly differentiated adenocarcinoma. TNM staging of tumor extension based on the new AICC/UICC classification (eighth edition) according to tumor site leads to a further improvement of the prognostic and therapeutic stratification of GEP-NETs and GEP-NECs.

The WHO classification of the pulmonary NENs distinguishes typical from atypical carcinoid and small cell carcinoma from large cell NECs (table 3). Necrosis and mitotic count are the main criteria used for the distinction of typical from atypical carcinoid, while small and large cell carcinomas are basically characterized by their cytological and histologic features.

The WHO classification of NENs of the head and neck distinguishes well-differentiated from moderately and poorly differentiated NECs. The distinction of these three tumor categories relies very much on the histologic features.

Other recent WHO classifications that concern NENs in the urogenital organs and the breast are either related to the categories of the GEP-NENs or the pulmonary NENs or both (table 3).

\section{Conclusions}

NENs have a common phenotype but vary in origin, morphology, function, molecular profile, type- and site-specific prognosis, aggressiveness, and response to treatment. The various currently available NEN classifications attempt to cope with these differences. Thus, all classifications recognize the existence of two major NEN families and provide a basis for their prognostic and therapeutic stratification. However, as the categorization of NENs can be improved by refined tumor grading, as it has been implemented in the current WHO classifications for gastrointestinal NENs and PanNENs from 2010 and 2017, a harmonization of the NEN classifications across organs should be pursued. A further improvement of the classifications can be expected by the inclusion of genetic data that could help to select tumors for targeted therapy.

\section{Disclosure Statement}

The author has no conflict of interest to declare.

\section{References}

1 Schimmack S, Svejda B, Lawrence B, Kidd M, Modlin IM: The diversity and commonalities of gastroenteropancreatic neuroendocrine tumors. Langenbecks Arch Surg 2011;396:273-298.

2 Gosset A, Masson P: Tumeurs endocrines de l'appendice. Presse Med 1914;25:237-240.

3 Feyrter F: Über diffuse endokrine epitheliale Organe. Leipzig, J.A. Barth, 1938.

4 Modlin IM, Champaneria MC, Bornschein J, Kidd M: Evolution of the diffuse neuroendocrine system - clear cells and cloudy origins. Neuroendocrinology 2006;84 69-82.

5 Pearse AG: The cytochemistry and ultrastructure of polypeptide hormone-producing cells of the APUD series and the embryologic, physiologic and pathologic implications of the concept. J Histochem Cytochem 1969;17:303-313.

6 Rosai J: The origin of neuroendocrine tumors and the neural crest saga. Mod Pathol 2011;24(suppl 2):S53-57.

7 Rindi G, Wiedenmann B: Neuroendocrine neoplasms of the gut and pancreas: new insights. Nat Rev Endocrinol 2011;8:54-64.

8 Pavel ME, Singh S, Strosberg JR, et al: Health-related quality of life for everolimus versus placebo in patients with advanced, non-functional, well-differentiated gastrointestinal or lung neuroendocrine tumours (RADIANT-4): a multicentre, randomised, double-blind, placebo-controlled, phase 3 trial. Lancet Oncol 2017; DOI: 10.1016/S1470-2045(17)30471-0.
9 Capella C, Heitz PU, Höfler H, Solcia E, Klöppel G: Revised classification of neuroendocrine tumours of the lung, pancreas and gut. Virchows Arch 1995;425: 547-560.

10 Solcia E, Klöppel G, Sobin L: Histological Typing of Endocrine Tumours, ed 2. Springer, Berlin, 2000.

11 DeLellis RA, Lloyd RV, Heitz PU, Eng C: Pathology and genetics of tumours of endocrine organs, ed 3. Lyon, IARC Press, 2004

12 Rindi G, Klöppel G, Ahlman H, et al: TNM staging of foregut (neuro)endocrine tumors: a consensus proposal including a grading system. Virchows Arch 2006; 449:395-401.

13 Rindi G, Klöppel G, Couvelard A, et al: TNM staging of midgut and hindgut (neuro)endocrine tumors: a consensus proposal including a grading system. Virchows Arch 2007;451:757-762.

14 Bosman FT, Carneiro F, Hruban RH, Theise ND (eds): WHO Classification of Tumours of the Digestive System, ed 4. Vol. 3. Lyon, IARC Press, 2010.

15 Lloyd RV, Osamura RY, Klöppel G, Rosai J: WHO Classification of Tumours of Endocrine Organs. Lyon, IARC Press, 2017.

16 Rindi G, Arnold R, Bosman F, et al: Nomenclature and classification of neuroendocrine neoplasms of the digestive system; in Bosman F, Carneiro F, Hruban R, Theise N (eds): WHO Classification of Tumours of the Digestive System. Lyon, IARC Press, 2010, pp 13-14.
17 Klimstra DS: Pathologic Classification of neuroendocrine neoplasms. Hematol Oncol Clin North Am 2016; 30:1-19.

18 Volante M, Brizzi MP, Faggiano A, et al: Somatostatin receptor type $2 \mathrm{~A}$ immunohistochemistry in neuroendocrine tumors: a proposal of scoring system correlated with somatostatin receptor scintigraphy. Mod Pathol 2007;20:1172-1182.

19 Konukiewitz B, Schlitter AM, Jesinghaus M, et al: Somatostatin receptor expression related to TP53 and $\mathrm{RB} 1$ alterations in pancreatic and extrapancreatic neuroendocrine neoplasms with a Ki67-index above 20 . Mod Pathol 2017;30:587-598.

20 Anlauf M, Schlenger R, Perren A, et al: Microadenomatosis of the endocrine pancreas in patients with and without the multiple endocrine neoplasia type 1 syndrome. Am J Surg Pathol 2006;30:560-574

21 Perigny M, Hammel P, Corcos O, et al: Pancreatic endocrine microadenomatosis in patients with von Hippel-Lindau disease: characterization by VHL/HIF pathway proteins expression. Am J Surg Pathol 2009; 33:739-748.

22 Jiao Y, Shi C, Edil BH, et al: DAXX/ATRX, MEN1, and mTOR pathway genes are frequently altered in pancreatic neuroendocrine tumors. Science 2011;331:11991203.

23 Yachida S, Vakiani E, White CM, et al: Small cell and large cell neuroendocrine carcinomas of the pancreas are genetically similar and distinct from well-differentiated pancreatic neuroendocrine tumors. Am J Surg Pathol 2012;36:173-184. 
24 Scarpa A, Chang DK, Nones K, et al: Whole-genome landscape of pancreatic neuroendocrine tumours. Nature 2017;543:65-71.

25 Marinoni I, Kurrer AS, Vassella E, et al: Loss of DAXX and ATRX are associated with chromosome instability and reduced survival of patients with pancreatic neuroendocrine tumors. Gastroenterology 2014;146:453460.e5.

26 Schmitt AM, Marinoni I, Blank A, Perren A: New genetics and genomic data on pancreatic neuroendocrine tumors: implications for diagnosis, treatment, and targeted therapies. Endocr Pathol 2016;27:200-204.

27 Agaimy A, Erlenbach-Wunsch K, Konukiewitz B, et al: ISL1 expression is not restricted to pancreatic well-differentiated neuroendocrine neoplasms, but is also commonly found in well and poorly differentiated neuroendocrine neoplasms of extrapancreatic origin. Mod Pathol 2013;26:995-1003.

28 Sorbye H, Strosberg J, Baudin E, Klimstra DS, Yao JC Gastroenteropancreatic high-grade neuroendocrine carcinoma. Cancer 2014;120:2814-2823.

29 Heetfeld M, Chougnet CN, Olsen IH, et al: Characteristics and treatment of patients with G3 gastroenteropancreatic neuroendocrine neoplasms. Endocr Relat Cancer 2015;22:657-664.
30 Hijioka S, Hosoda W, Matsuo K, et al: Rb loss and KRAS mutation are predictors of the response to platinum-based chemotherapy in pancreatic neuroendocrine neoplasm with grade 3: a Japanese multicenter pancreatic NEN-G3 study. Clin Cancer Res 2017;23: $4625-4632$.

31 Swarts DR, Ramaekers FC, Speel EJ: Molecular and cellular biology of neuroendocrine lung tumors: evidence for separate biological entities. Biochim Biophys Acta 2012;1826:255-271.

32 Schonhoff SE, Giel-Moloney M, Leiter AB: Minireview: development and differentiation of gut endocrine cells. Endocrinology 2004;145:2639-2644.

33 van Eeden S, Offerhaus GJ: Historical, current and future perspectives on gastrointestinal and pancreatic endocrine tumors. Virchows Arch 2006;448:1-6.

34 Hermann G, Konukiewitz B, Schmitt A, Perren A, Klöppel G: Hormonally defined pancreatic and duodenal neuroendocrine tumors differ in their transcription factor signatures: expression of ISL1, PDX1, NGN3, and CDX2. Virchows Arch 2011;459:147-154.

35 Anlauf M, Perren A, Henopp T, et al: Allelic deletion of the MEN1 gene in duodenal gastrin and somatostatin cell neoplasms and their precursor lesions. Gut 2007;56:637-644.
36 Anlauf M, Perren A, Klöppel G: Endocrine precursor lesions and microadenomas of the duodenum and pancreas with and without MEN1: criteria, molecular concepts and clinical significance. Pathobiology 2007; 74:279-284.

37 Perren A, Anlauf M, Henopp T, et al: Multiple endocrine neoplasia type 1: loss of one MEN1 allele in tumors and monohormonal endocrine cell clusters, but not in islet hyperplasia of the pancreas. A combined FISH and immunofluorescence study. J Clin Endocrinol Metab 2007;92:1118-1128.

38 Pipeleers-Marichal M, Somers G, Willems G, et al: Gastrinomas in the duodenums of patients with multiple endocrine neoplasia type 1 and the Zollinger-Ellison syndrome. N Engl J Med 1990;322:723-727.

39 Klöppel G, Anlauf M, Perren A, Sipos B: Hyperplasia to neoplasia sequence of duodenal and pancreatic neuroendocrine diseases and pseudohyperplasia of the PPcells in the pancreas. Endocr Pathol 2014;25:181-185.

40 Nieser M, Henopp T, Brix J, et al: Loss of chromosome 18 in neuroendocrine tumors of the small intestine: the enigma remains. Neuroendocrinology 2017;104:302312

41 Rindi G, Falconi M, Klersy C, et al: TNM staging of neoplasms of the endocrine pancreas: results from a large international cohort study. J Natl Cancer Inst 2012;104:764-777. 